\title{
Meta
}

Journal des traducteurs

Translators' Journal

\section{VARElA SAlinAs, MARÍA JosÉ, dir. (2009) : Panorama actual del estudio y la enseñanza de discursos especializados. Bern : Peter Lang, 277 p.}

\section{Mariana Raffo}

Volume 57, numéro 1, mars 2012

URI : https://id.erudit.org/iderudit/1012755ar

DOI : https://doi.org/10.7202/1012755ar

Aller au sommaire du numéro

Éditeur(s)

Les Presses de l’Université de Montréal

ISSN

0026-0452 (imprimé)

1492-1421 (numérique)

Découvrir la revue

Citer ce compte rendu

Raffo, M. (2012). Compte rendu de [VARELA SALINAs, MARía JosÉ, dir. (2009) : Panorama actual del estudio y la enseñanza de discursos especializados. Bern :

Peter Lang, 277 p.] Meta, 57(1), 267-269. https://doi.org/10.7202/1012755ar

Ce document est protégé par la loi sur le droit d'auteur. L'utilisation des services d’Érudit (y compris la reproduction) est assujettie à sa politique d'utilisation que vous pouvez consulter en ligne.

https://apropos.erudit.org/fr/usagers/politique-dutilisation/
Cet article est diffusé et préservé par Érudit.

Érudit est un consortium interuniversitaire sans but lucratif composé de l’Université de Montréal, l'Université Laval et l'Université du Québec à Montréal. Il a pour mission la promotion et la valorisation de la recherche. https://www.erudit.org/fr/ 
culturelle du traducteur en se centrant sur le cas de la traduction vers la langue et la culture arabes, dans des sociétés majoritairement musulmanes, dans le contexte actuel de mondialisation. Dima El Husseini dans «Retour aux sources et recréation de l'effet» analyse un cas spécial de la traduction littéraire: la traduction en arabe d'un roman d'une auteure égyptienne d'expression française; sa particularité réside dans le fait qu'il s'agit d'une traduction qui ramène l'œuvre à sa culture d'origine.

Cette deuxième partie s'achève avec «Pour une sémiotique de la traduction» de Sündüz Öztürk Kasar, qui envisage l'intérêt d'appliquer la méthode sémiotique, et plus concrètement la sémiotique littéraire, à l'étude de la traduction; elle propose ainsi une sémio-analytique de la traduction, un modèle de lecture et d'analyse applicable à trois étapes de la traduction: le discours (le texte littéraire), l'interdiscours (la révision) et le métadiscours (la critique ou l'analyse du texte traduit).

La troisième partie, "Enseignement de la traduction et de l'interprétation", recueille sept contributions d'orientation pédagogique, les unes d'ordre plus général sur les bases théoriques et l'orientation de la formation, d'autres en rapport avec l'enseignement de matières spécifiques (la traduction spécialisée, l'interprétation).

Don Kiraly dans «Acknowledging Learning as Enaction Moving beyond Social Constructivism towards Empowerment in Translator Education" passe en revue un ensemble de concepts pédagogiques-clés et complémentaires pour une nouvelle pédagogie de la traduction: l'apprentissage comme acquisition (approche acquisitive/transmissive de l'enseignement), l'apprentissage comme processus réflexif sociocognitif (apprentissage coopératif), l'apprentissage comme processus basé sur la pratique de participation à des communautés, et l'apprentissage comme co-émergence. Dans «La traductologie et le cours de traduction ", Christian Balliu présente le rôle de la traductologie dans les cours de traduction, propose un enseignement de la traductologie appliquée et expose les raisons de l'«exil théorique» qui se produit parfois dans les cours de traduction. "Translation Training in Italy. An Analysis of Students' Perceptions » de Sabrina Fusari présente les résultats d'un questionnaire destiné à des étudiants sur les objectifs professionnels poursuivis et la perception de l'offre éducative en traduction et interprétation.

Nicolas Froeliger dans «À quoi bon enseigner la traduction technique» se demande comment délimiter et définir le domaine de la traduction technique et comment structurer son enseignement. «Animer un atelier de traduction spécialisée. Une expérience en chantier» de Freddie Plassard présente l'expérience d'un atelier de traduction spécialisée en salle d'informatique multiposte et expose le cadre pédagogique (compétence de traduction, exercices, évaluation, etc.) ainsi que les écueils auxquels il faut faire face (choix des textes, assimilation des connaissances notionnelles, etc.). «Les phases critiques de la formation en interprétation. Approches didactiques» de Karla Déjean Le Féal met l'accent sur l'importance de la progression dans la formation des interprètes; de ce point de vue, l'auteure part de la conception de quatre phases-clés dans l'apprentissage de l'interprétation et propose une approche pour chacune d'elles: le tout début de la formation, le début de la prise de notes, la préparation du passage à l'interprétation simultanée (la traduction à vue) et l'initiation à l'interprétation simultanée. Enfin, «L'enseignement de l'interprétation. Principe universel et connaissances spécifiques» de Hiromi Ito-Bergerot analyse les caractéristiques spécifiques de l'apprentissage de l'interprétation consécutive japonais-français à partir de l'enregistrement des cours et des notes des étudiants pendant une année universitaire; les résultats le mènent à conclure que l'acquisition de la méthode interprétative peut s'accompagner d'un approfondissement des connaissances linguistiques propres à chaque combinaison linguistique.

La diversité des thèmes exposés et des points de vue rend compte du grand nombre d'objets d'étude ainsi que de la richesse théorique dont jouit actuellement la traductologie, de même que des perspectives de recherches qui s'ouvrent, ce que reflète très bien le titre de la deuxième partie de l'ouvrage, Horizons traductologiques.

Amparo Hurtado Albir Universitat Autònoma de Barcelona, Barcelone, Espagne

VArela Salinas, María José, dir. (2009): Panorama actual del estudio y la enseñanza de discursos especializados. Bern: Peter Lang, $277 \mathrm{p}$.

Contrairement à ce que suggère le titre, Panorama actuel de l'étude et de l'enseignement des discours spécialisés ${ }^{1}$, ce collectif est presque entièrement consacré à la traduction spécialisée. De fait, les neuf chapitres -rédigés en espagnol à l'exception $\mathrm{du}$ dernier, en allemand- sont repartis en trois sections: traduction juridique, traduction biomédicale et thèmes divers. La plupart des articles abordent le discours d'un domaine de spécialité à partir des problématiques posées par la pratique ou par la pédagogie de la traduction. Certains se penchent sur des paires de langues ou des contextes culturels spécifiques tandis que d'autres traitent de questions plus générales. La directrice, María José 
Varela Salinas, explicite toutefois la vocation large du volume: "Il s'agit de travaux qui proposent de nouvelles approches pour la pédagogie des langues de spécialité, soit par des applications concrètes, soit à l'aide d'une réflexion théorique ${ }^{2} »($ p. 7).

La première partie, axée sur le droit, comprend trois analyses comparatives dont une étude terminologique. Dans le premier chapitre, Inmaculada Almahano Güeto caractérise trois types de contrats touristiques utilisés en Espagne et en Allemagne et les situe dans les typologies élaborées pour les textes juridiques. Elle met en relief l'hétérogénéité des taxonomies existantes, qui se fondent sur des principes de classement assez divers, et fait état des difficultés pour cerner la classe des «contrats » à l'aide de ces critères, car elle se trouve parfois à cheval entre deux catégories. Il en résulte une description exhaustive des aspects communicatifs, pragmatiques et linguistiques, ainsi que des deux cadres institutionnels où les textes apparaissent, analyse qui s'avère cruciale au moment de la traduction. Le deuxième chapitre, écrit par Adela Martínez García, traite des écueils que représentent pour les traducteurs novices les asymétries culturelles et linguistiques entre deux régimes juridiques: la Common Law anglo-galloise et le droit espagnol. De fait, la prise de décisions de traduction doit tenir compte des circonstances historiques et sociales qui en ont marqué la naissance. L'auteure explique la démarche suivie pour introduire les notions essentielles dans le cadre d'un cours de traduction juridique et évoque les stratégies à adopter lorsque l'équivalence fonctionnelle fait défaut. Dans le troisième chapitre, Encarnación Tabares Plasencia et Vessela Ivanova abordent la variation géographique dans le discours juridique, d'autant plus saillante dans un domaine ayant une forte spécificité socio-culturelle. Après une révision détaillée des débats terminologiques actuels et des travaux sur la variation, les auteures constatent que les ressources terminographiques multilingues ne gèrent pas toujours correctement ce phénomène et que la documentation demeure indispensable pour les traducteurs. La problématique est illustrée par une étude d'envergure portant sur la paire allemand-espagnol: la variation terminologique et conceptuelle - intra et interlinguistique - liée à la notion de meurtre est examinée au sein de neuf systèmes juridiques différents. La difficulté qui en découle pour l'établissement de l'équivalence impose, lors de la traduction, de situer les unités terminologiques dans leur cadre juridique spécifique.

La deuxième partie cible la formation des traducteurs et la pratique dans le domaine biomédical. María Calonge Prieto attire l'attention sur certaines particularités de la langue médicale qui posent des difficultés aux apprentis-traducteurs, et tout particulièrement sur la variation. Autant la connaissance du champ notionnel que la compréhension des motivations sociales, linguistiques et contextuelles qui sous-tendent l'usage des termes sont des facteurs clés pour éviter les erreurs. Il est donc d'une grande utilité pour les étudiants de confectionner des fiches terminologiques à partir de corpus authentiques: cette tâche leur permet d'étoffer leur bagage encyclopédique et de situer les termes dans leur contexte d'emploi. Pour leur part, Ana Isabel García Esteban et María José Varela Salinas présentent la méthode pédagogique conçue pour le cours de traduction spécialisée allemandespagnol à l'Universidad de Málaga. Le but des séances consacrées à la traduction biomédicale est de sensibiliser les étudiants à la diversité du discours spécialisé en introduisant des genres de textes variés, choisis en fonction des besoins du marché du travail en Espagne. Conformément au cadre fonctionnaliste adopté, une grande importance est accordée aux paramètres de la consigne de traduction. Les auteures décrivent en détail le déroulement de la première unité didactique et les critères adoptés pour l'évaluation. Dans le dernier article de cette section, Laura Parrilla Gómez se penche sur les ressources multilingues qui sont de plus en plus utilisées en milieu clinique afin de faciliter la communication entre le personnel soignant et les patients. À partir de l'analyse de plusieurs guides plurilingues, elle passe en revue les aspects à considérer lors de la création de ces textes, du sujet choisi aux modalités de diffusion. Le travail conjoint des médecins et des traducteurs est fondamental pour la mise au point d'un outil efficace et adéquat sur les plans communicatif, notamment en ce qui concerne le niveau de spécialisation, et culturel.

La troisième partie regroupe des travaux assez divers. Les deux premiers semblent même échapper en quelque sorte à la thématique du volume, car il n'y est pas strictement question de traduction spécialisée. Ainsi, Isabel Comitre Narváez adapte l'approche descriptive à l'étude de la traduction audiovisuelle, où la composante sémiotique est capitale. Elle compare deux traductions du film Peau d'Âne de J. Demy afin de dégager des normes régissant la «retraduction» d'une œuvre cinématographique. Si le premier doublage reste proche de l'original, la seconde version (doublage et sous-titrage) présente plusieurs instances de manipulation visant l'"acceptabilité» du texte dans la culture cible. Le chapitre suivant, rédigé par Rosario Herrero Prádanos, porte sur le discours d'un domaine peu étudié: celui des revues de mode. L'analyse permet de montrer que la «langue de la mode» peut être considérée comme une langue de spécialité à part entière, car elle possède une terminologie et des ressources stylistiques 
particulières. Ici aussi la dimension sémiotique s'avère fondamentale. Enfin, Karl-Heinz Freigang, Yamile Ramírez et Daniel Zielinski proposent une méthodologie d'enseignement de la traduction spécialisée faisant une place accrue aux outils informatiques. Ils prônent une approche pédagogique "collaborative» simulant une commande réelle où tous les acteurs et les étapes du projet sont pris en compte afin d'encourager le travail en équipe. Aussi, l'emploi des logiciels liés aux différents tâches (gestion de projet, recherche terminologique, traduction et révision) est intégré au travail en classe.

De prime abord, il semble étonnant que ce collectif soit présenté comme un «échantillon représentatif» des recherches actuelles sur l'enseignement des discours spécialisés (p. 7), à moins que l'on restreigne ce champ d'études à la pédagogie de la traduction. Nous avons, à défaut, un aperçu des travaux menés aujourd'hui en Espagne sur la traduction spécialisée. Dans ce sens, le volume constitue un apport indéniable, ne serait-ce que par la diversité des approches et des sujets traités ${ }^{3}$. Certes, on peut critiquer un manque de rigueur dans la démarche ou dans la présentation de certaines analyses, et quelques coquilles et erreurs d'édition sont à déplorer (par exemple, la fin du deuxième chapitre semble tronquée). Il reste que les questions soulevées sont pertinentes pour la pratique et l'enseignement de la traduction spécialisée et que les problématiques sont envisagées dans une optique discursive, en tenant compte des aspects communicatifs et textuels aussi bien que linguistiques. Par ailleurs, nous ne pouvons que saluer la publication d'un recueil d'études sur la traduction qui s'appuient, pour la plupart, sur des données authentiques.

Mariana RafFo Université de Montréal, Montréal, Canada

\section{NOTES}

1. Notre traduction.

2. Notre traduction.

3. Il faut toutefois signaler l'absence de travaux sous l'angle cognitif.

Regattin, Fabio (2009): Le jeu des mots. Bologne: I libri di Emil, 159 p.

Tout comme la poésie, les jeux de mots ont très souvent été donnés en exemple d'intraduisibilité par les partisans de l'objection préjudicielle. Pourtant, bien qu'ils soient rares, certains auteurs intéressés par la question ont démontré aisément que le transfert de différents jeux linguistiques est possible (nous pensons notamment à Jacqueline Henry et son remarquable ouvrage La traduction des jeux de mots, publié en 2003). Il en reste beaucoup à dire sur ce défi traductionnel encore trop souvent négligé, et avec Le jeu des mots, Fabio Regattin apporte une contribution importante à la réflexion sur ce domaine complexe. D'abord traducteur théâtral et littéraire, ce docteur en traductologie s'intéresse particulièrement à la traduction culturelle et nul doute que son expérience de praticien a influencé ses travaux. Ainsi, s'inspirant des propos de James Holmes sur l'importance de développer en traductologie des théories qui portent sur des problèmes précis, Regattin s'est donné comme objectif d'élaborer une théorie unique des jeux de mots. Il serait alors possible non seulement de comparer un jeu de mots et ses différentes traductions, mais également d'évaluer ces dernières objectivement.

Pour parvenir à cette fin, la première étape est naturellement de retenir une définition universelle de la notion quelque peu fourre-tout de jeu. Regattin relève donc dans le premier chapitre plusieurs définitions provenant de sources variées et remarque qu'elles ne sont que partielles. Il avance que les nombreux types de jeux démontrent tant un aspect gratuit et libre qu'un aspect structuré et réglé, et c'est précisément cette distinction pourtant facilement observable qui manque aux définitions citées par l'auteur. L'aspect libre et gratuit est représenté par le mot anglais play alors que la notion de jeu structuré est partagée entre les mots anglais game (la langue dans le cas des jeux de mots) et play (la parole). En d'autres termes, Regattin suggère de distinguer le jeu gratuit, le jeu tel que défini par ses règles et le jeu en tant que réalisation de la règle en question. Cette triple division, tout à fait logique mais quelque peu complexe au premier regard (d'autant plus que l'auteur ne donne aucun exemple), est le noyau de l'ouvrage. L'utilisation de ce modèle, tant pour approfondir la réflexion théorique que pour analyser des exemples pratiques de traduction de jeux de mots, constitue le principal apport de Jeu des mots à la traductologie.

Dans le second chapitre, Regattin continue d'établir le cadre théorique de sa réflexion en s'intéressant cette fois spécifiquement à la notion de jeu de mots. Il présente encore une fois une série de définitions différentes avant d'en extraire quelques points communs, notamment leur aspect formel, leur longueur réduite et la présence d'une certaine ambiguïté. L'auteur poursuit en présentant divers systèmes de classification des jeux de mots, en axant soit sur la linguistique, sur les composantes formelles ou encore sur les effets psychologiques recherchés. Ce survol de la question, assez long vu la dimension de l'ouvrage mais somme toute pertinent, lui permet de concevoir sa propre définition: «Un texte aux dimensions variables, qui implique 